\title{
评 述＜wide>原子核协变密度泛函理论及其应用专辑
}

\section{多准粒子组态的手征转动模型}

\author{
开斌*，贾慧
}

山东大学空间科学研究院, 山东大学 (威海) 空间科学与物理学院, 山东省光学天文与日地空间环境重点实验室, 威海 264209

*联系人, E-mail: bqi@sdu.edu.cn

收稿日期: 2015-07-15; 接受日期: 2015-08-25; 网络出版日期: 2015-12-14

国家自然科学基金资助项目 (批准号: 11405096, 1146114100)

\begin{abstract}
摘要原子核的手征转动现象近年来引起了广泛的关注. 本文将介绍两种用于分析多准粒子组态手征转动 的模型, 即粒子转子模型和倾斜轴推转模型. 对于粒子转子模型, 主要介绍基矢的构造和模型的求解: 通过选 取价核子单粒子态及角动量量子数的限制条件来构造严格满足 $D_{2}$ 对称性的强耦合基矢; 当考虑多个价核子 时, 模型的空间维数巨大, 通过 Lancozos 对角化方法来求解大规模哈密顿量矩阵. 对于倾斜轴推转模型, 分别 介绍了基于对力加四极相互作用和基于壳修正方法的两种版本: 由转动参考系下的两体 Routhian 量出发给出 推转的 Hartree-Fock-Bogoliubov 方程; 在内禀的主轴系中分析了三维倾斜轴转动的自洽解, 此时角速度矢量与角 动量期待值矢量平行. 通过总结和进一步发展两种理论模型, 将为澄清原子核手征转动研究中的种种争论奠 定基础.
\end{abstract}

关键词手征转动, 粒子转子模型, 倾斜轴推转模型, 多准粒子组态

PACS: 21.60.Ev, 21.10.Re, 23.20.Lv

\section{1 引言}

1997 年，原子核的手征性被 Frauendorf 和 Meng 预言 ${ }^{11]}$ : 考虑一个三轴形变的原子核, 其 价质子和价中子处于高 $-j$ 壳上, 分别具有粒子 性和空穴性. 为了使得整个转动体系的能量最小, 粒子性的价质子、空穴性的价中子和由其余核 子所组成的核芯的角动量 $\boldsymbol{j}_{p}, \boldsymbol{j}_{n}$ 和 $\boldsymbol{R}$ 将分别沿 原子核的三个惯性主轴排列。 三个互相垂直的 角动量可以形成两种具有相反手性的体系, 即左 手系和右手系, 于是本体系下发生了手征对称性
的自发破缺. 通过手征变换 $\chi=\mathscr{T} \mathscr{R}(\pi)$ 可以使得左 手系和右手系进行相互转换. 而左手系和右手系之 间的量子隧穿效应, 使得能够从实验上观察到原子 核的手征对称性破缺 一 近简并的两条 $\Delta I=1$ 的转 动带, 即手征双重带.

自从手征双重带被预言以后, 引起了各大实验 室的探索热情. 2001 年在 $N=75$ 的同中子素中观 测到一系列候选的手征双重带 ${ }^{[2]}$. 随后, 在质量 数 $\mathrm{A} \sim 130^{[3-18]}$ 和 $\mathrm{A} \sim 100^{[19-28]}$ 区报道了近 30 例候 选的手征双重带, 形成两个 “手征核岛”。而在 A $190^{[29,30]}$ 和 $\mathrm{A} \sim 80^{[31]}$ 质量区, 也各报道了一两例候选

引用格式: 元斌, 贾慧. 多准粒子组态的手征转动模型. 中国科学: 物理学 力学 天文学, 2016, 46: 012014

Qi B, Jia H. Chiral rotational model with multi-quasi-particle configuration (in Chinese). Sci Sin-Phys Mech Astron, 2016, 46: 012014, doi: 10.1360/SSPMA2015-00362 
手征核, 能否形成新的“手征核岛” 需要进一步的实 验探索. 为了确认原子核手性的存在, 除了观测到 近简并的转动带之外, 电磁跃迁性质的测量也非常 重要. 2006 年首先对候选手征核 ${ }^{134} \mathrm{Pr}$ 进行了能级寿 命测量, 提取了电四极跃迁和磁偶极跃迁几率 [32,33], 似乎并不符合手征带所期待的电磁跃迁信号 [34]. 而 随后 ${ }^{128} \mathrm{Cs}^{[35]},{ }^{135} \mathrm{Nd}^{[36]}$ 的实验结果则非常符合手 征带所期待的特征. 近期对 ${ }^{126} \mathrm{Cs}{ }^{[37]},{ }^{102} \mathrm{Rh}^{[38]}$ 和 ${ }^{106} \mathrm{Ag}{ }^{[39,40]}$ 等核也进行了能级寿命测量. 多手征带 $(\mathrm{M} \chi \mathrm{D})$ 是近两年实验组关注的又一有趣现象, 这一概 念最早由理论所预言 ${ }^{[41-44]}$, 即在一个原子核中可能 存在多对手征双重带. 在 2013 年第一个多手征带的

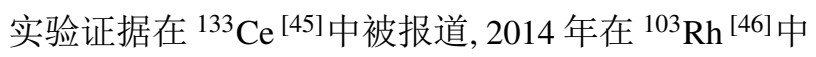
也报道了 $\mathrm{M} \chi \mathrm{D}$ 的证据. 关于原子核手征转动研究进 展的较全面的总结可以参考文献 [47-51].

在理论研究方面, 手征双重带的最初提出, 源于倾斜轴推转模型(TAC) 近似和粒子转子模型 $(\mathrm{PRM})^{[1]}$. 之后很多努力致力于发展这两种模型来 研究实际核中的手征双重带. 此外, 相互作用玻色子 模型 ${ }^{[32]}$, 投影壳模型 ${ }^{[52]}$ 和集体哈密顿量 ${ }^{[53]}$ 也用于 描述原子核的手征转动. 最初采用的三维倾斜轴推 转模型基于单 $j$ 壳模型 ${ }^{[1]}$, 之后发展为基于 Woods-

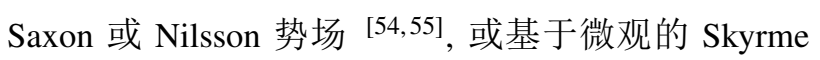
Hartree Fock 模型 ${ }^{[56,57]}$. 二维倾斜轴推转的相对论 平均场模型也已经成功地描述了磁转动和反磁转动 等 ${ }^{[42,58-62]}$, 但三维倾斜轴推转的版本仍在发展之中. 倾斜轴推转模型的优点在于能对原子核的转动现象 做完全微观的描述. 但是, 平均场近似破坏了转动不 变性, 因此其角动量并不是好量子数. 作为一个半经 典理论, 它只能给出原子核是否存在倾斜转动, 并不 能给出双带. 想要计算双带的能谱结构, 需要超越平 均场, 如考虑无规相位近似等 ${ }^{[36]}$; 此外计算电磁跃迁 在此模型中也采用半经典的近似处理.

作为一个量子化的模型, 粒子转子模型也广泛 应用于手征双重带的理论分析. 一粒子一空穴的粒 子转子模型常用于分析理想手征带的性质 [63-65]. 考 虑对关联后, 准质子准中子与三轴转子耦合的模型对 奇奇核的手征双重带提供了较好的描述 ${ }^{[35,66-69]}$. 但 是对于奇 $\mathrm{A}$ 核中的手征带, 它主要建立在三准粒子 组态之上, 而对于偶偶核, 可能建立基于四准粒子组
态的手征带. 为此, 2009 年文献 [70]建立了多粒子多 空穴与三轴转子耦合的模型, 并用于处理涉及多个 价核子组态的原子核的手征转动现象 ${ }^{[40,46,71-73]}$. 粒 子转子模型中, 总角动量是好量子数. 能量和跃迁几 率被完全量子化地处理. 特别地, 在整个自旋范围内, 即遍及手征振动到静态手性区域, 它可以给出双带间 的能量䢃裂. 不过这一模型是一个唯象的模型, 转子 的形变以及价核子运动的势场是唯象给定的. 此外, 当处理多个价核子时, 基矢维数巨大, 计算耗时.

倾斜轴推转模型和粒子转子模型是目前用于分 析手征转动的两种主要模型, 两者都有其各自的优 缺点, 在分析原子核手征转动时需要互相补充. 本文 将介绍这两种用于分析手征转动的模型. 我们将在 多准粒子组态下介绍理论框架, 从而能够用于各种 组态的候选手征双重带的理论分析.

\section{2 多准粒子组态的粒子转子模型}

为了描述单粒子运动和集体运动之间的相互影 响, Bohr 和 Mottelson ${ }^{[74]}$ 建议考虑几个所谓的价核 子, 它们在核芯的形变势场中近似独立地运动, 将价 核子与代表所有剩余核子的集体转子进行耦合, 这 就是粒子转子模型 (PRM). 一般地, 将 PRM 哈密顿量 分为两部分:

$$
\hat{H}=\hat{H}_{\text {coll }}+\hat{H}_{\text {intr }} \text {. }
$$

唯象的集体部分 $H_{\text {coll }}$ 描述惰性的核芯, 微观的内禀 部分 $H_{\text {intr }}$ 描述一个价核子或几个价核子, 乃至费米 能附近整个子壳上的核子 [75].

\section{1 模型基矢}

为了表述方便, 这里我们考虑单 $-j$ 壳模型, 价核 子的单粒子态为

$$
\begin{aligned}
& a_{v}^{+}|0\rangle=\sum_{\Omega} c_{\Omega}^{(v)}|j, \Omega\rangle, \\
& a_{\bar{v}}^{+}|0\rangle=\sum_{\Omega}(-1)^{j-\Omega} c_{\Omega}^{(v)}|j,-\Omega\rangle,
\end{aligned}
$$

其中 $\Omega$ 是单粒子角动量 $\hat{j}$ 沿 3- 轴的投影, 由于时 间反演对称性的存在, $\Omega$ 取值通常为 $\cdots,-7 / 2,-3 / 2$, $+1 / 2,+5 / 2, \cdots$. 将单粒子态分成互为时间反演态的 
两个子空间是必要的, 这样取值能够在构造 PRM 基 矢时考虑满足 $D_{2}\left(\hat{R}_{1}(\pi), \hat{R}_{2}(\pi), \hat{R}_{3}(\pi)\right)$ 对称性的形式.

于是对于具有 $z$ 个价质子和 $n$ 个价中子的体系, 内禀波函数为

$$
\begin{aligned}
|\varphi\rangle & =\left|\varphi_{p}\right\rangle\left|\varphi_{n}\right\rangle \\
& =\left(\prod_{i=1}^{z_{1}} a_{p, v_{i}}^{\dagger}\right)\left(\prod_{i=1}^{z_{2}} a_{p, \overline{\mu_{i}}}^{\dagger}\right)\left(\prod_{i=1}^{n_{1}} a_{n, v_{i}^{\prime}}^{\dagger}\right)\left(\prod_{i=1}^{n_{2}} a_{n, \overline{\mu_{i}^{\prime}}}^{\dagger}\right)|0\rangle
\end{aligned}
$$

其中 $z_{1}+z_{2}=z, n_{1}+n_{2}=n, 0 \leqslant z_{1} \leqslant z, 0 \leqslant n_{1} \leqslant n$. $a_{p, v_{1}}^{\dagger}|0\rangle, \quad \ldots, \quad a_{p, v_{z_{1}}}^{\dagger}|0\rangle, \quad a_{p, \bar{\mu}_{1}}^{\dagger}|0\rangle, \ldots, \quad a_{p, \bar{\mu}_{z_{2}}}^{\dagger}|0\rangle$ 是从 $2 j_{p}+1$ 个单质子态中选取的任意的 $z$ 个态, 中子情况 类似.

我们构造强耦合基矢为

$$
\begin{aligned}
|I M K \varphi\rangle= & \frac{1}{\sqrt{2\left(1+\delta_{K 0} \delta_{\varphi, \bar{\varphi}}\right.}} \\
& \times\left(|I M K\rangle|\varphi\rangle+(-1)^{I-K}|I M-K\rangle|\bar{\varphi}\rangle\right),
\end{aligned}
$$

其中 $|\bar{\varphi}\rangle$ 为

$$
\begin{aligned}
|\bar{\varphi}\rangle= & \hat{R}_{2}(\pi)|\varphi\rangle \\
= & (-1)^{z_{2}}(-1)^{n_{2}}\left(\prod_{i=1}^{z_{1}} a_{p, \overline{v_{i}}}^{\dagger}\right)\left(\prod_{i=1}^{z_{2}} a_{p, \mu_{i}}^{\dagger}\right) \\
& \times\left(\prod_{i=1}^{n_{1}} a_{n, \overline{v_{i}^{\prime}}}^{\dagger}\right)\left(\prod_{i=1}^{n_{2}} a_{n, \mu_{i}^{\prime}}^{\dagger}\right)|0\rangle \\
= & (-1)^{z_{2}}(-1)^{z_{1} z_{2}}\left(\prod_{i=1}^{z_{2}} a_{p, \mu_{i}}^{\dagger}\right)\left(\prod_{i=1}^{z_{1}} a_{p, \overline{v_{i}}}^{\dagger}\right) \\
& \times(-1)^{n_{2}}(-1)^{n_{1} n_{2}}\left(\prod_{i=1}^{n_{2}} a_{n, \mu_{i}^{\prime}}^{\dagger}\right)\left(\prod_{i=1}^{n_{1}} a_{n, \overline{v_{i}^{\prime}}}^{\dagger}\right)|0\rangle,
\end{aligned}
$$

式中出现的相因子 $(-1)^{z_{2}},(-1)^{n_{2}}$ 源自每个单粒子态 转动 $2 \pi$ 后会出现的因子, 即

$$
\hat{R}_{2}(\pi)^{2} a_{v}^{+}|0\rangle=-a_{v}^{+}|0\rangle .
$$

如上构造的基矢满足 $\hat{R}_{2}(\pi)$ 对称性, 为避免基矢 被重复考虑, 需要对 $z_{1}, z_{2}, n_{1}, n_{2}$ 的取值做限制. 进一 步要求基矢满足 $\hat{R}_{3}(\pi)$ 对称性, 这将限制 $K-\frac{1}{2}\left(z_{1}-\right.$ $\left.z_{2}\right)-\frac{1}{2}\left(n_{1}-n_{2}\right)$ 为偶数. 这样, 构造的基矢最终就满 足了 $D_{2}$ 对称性.
对于一个确定的自旋 $I$, 当采用单 $-j$ 壳模型描述 价核子时, PRM 基矢空间的维数将是

$$
C_{2 j_{p}+1}^{z} C_{2 j_{n}+1}^{n}(2 I+1) / 4 .
$$

第一个因子是从 $2 j_{p}+1$ 个单质子态中任意选取 $z$ 个 态的组态数目, 第二个因子来自中子态的选取. 因子 $2 I+1$ 是 $K$ 的可能取值数目, 因子 $1 / 4$ 来自基矢满足 $D_{2}$ 对称性的限制. 当考虑较多价核子组态时, 模型基 矢维数会过大. 这时, 可以采用多粒子组态能量截断 方案, 即只考虑满足价核子的激发能量之和小于某 一截断能量值这一条件的组态作为基矢, 以有效减 少基矢维数.

\section{2 模型的求解}

下面在这样一组对称化的强耦合基上来构建哈 密顿量矩阵. 由于选取的基矢中包含着单粒子本征 态, 因此内禀部分的哈密顿量, 其非零的矩阵元只在 对角项存在, 是容易给出的.

由于基矢中涉及全同的多个价质子或价中子体 系, 因此对于集体部分哈密顿量

$$
\hat{H}_{\text {coll }}=\sum_{i=1}^{3} \frac{\hat{R}_{i}^{2}}{2 \mathscr{J}_{i}}=\sum_{i=1}^{3} \frac{\hat{I}_{i}^{2}}{2 \mathscr{J}_{i}}-\sum_{i=1}^{3} \frac{\hat{I}_{i} \hat{J}_{i}}{\mathscr{J}_{i}}+\sum_{i=1}^{3} \frac{\hat{J}_{i}^{2}}{2 \mathscr{J}_{i}},
$$

采用二次量子化形式是方便的. 角动量 $\hat{J}_{\alpha}$ (代表 $\hat{J}_{+}$, $\left.\hat{J}_{-}, \hat{J}_{3}\right)$ 为单体算符,

$$
\hat{J}_{\alpha}=\sum_{\tau_{3}} \sum_{\mu v}\left\langle\hat{j}_{\alpha}\right\rangle_{\mu v} a_{\tau_{3}, \mu}^{+} a_{\tau_{3}, v},
$$

其中 $\tau_{3}$ 为同位旋标记, $\tau_{3}=1 / 2$ 代表质子, $\tau_{3}=-1 / 2$ 代表中子. 角动量平方项 $\hat{J}_{\alpha}^{2}$ (代表 $\hat{J}_{+}^{2}, \hat{J}_{-}^{2}, \hat{J}_{3}^{2}$ ) 中包含 单体算符和两体算符:

$$
\begin{aligned}
\hat{J}_{\alpha}^{2}= & \sum_{\tau_{3}, \tau_{3}^{\prime}} \sum_{\mu \nu \mu^{\prime} v^{\prime}}\left\langle j_{\tau_{3}, \alpha}\right\rangle_{\mu v} a_{\tau_{3}, \mu}^{+} a_{\tau_{3}, v}\left\langle j_{\tau_{3}^{\prime}, \alpha}\right\rangle_{\mu^{\prime} v^{\prime}} a_{\tau_{3}^{\prime}, \mu^{\prime}}^{+} a_{\tau_{3}^{\prime}, v^{\prime}} \\
= & \sum_{\tau_{3}} \sum_{\mu v}\left\langle j_{\tau_{3}, \alpha}^{2}\right\rangle_{\mu v} a_{\tau_{3}, \mu}^{+} a_{\tau_{3}, v} \\
& +\sum_{\tau_{3} \neq \tau_{3}^{\prime}} \sum_{\mu v}\left\langle j_{\tau_{3}, \alpha}\right\rangle_{\mu v} a_{\tau_{3}, \mu}^{+} a_{\tau_{3}, v}\left\langle j_{\tau_{3}^{\prime}, \alpha}\right\rangle_{\mu^{\prime} v^{\prime}} a_{\tau_{3}^{\prime}, \mu^{\prime}}^{+} a_{\tau_{3}^{\prime}, v^{\prime}} \\
& +2 \sum_{\tau_{3}} \sum_{v<v^{\prime}, \mu<\mu^{\prime}}\left(\left\langle j_{\tau_{3}, \alpha}\right\rangle_{\mu v}\left\langle j_{\tau_{3}, \alpha}\right\rangle_{\mu^{\prime} v^{\prime}}\right. \\
& \left.-\left\langle j_{\tau_{3}, \alpha}\right\rangle_{\mu^{\prime} v}\left\langle j_{\tau_{3}, \alpha}\right\rangle_{\mu v^{\prime}}\right) a_{\tau_{\tau_{3}, \mu}}^{+} a_{\tau_{3}, v} a_{\tau_{3}, \mu^{\prime}}^{+} a_{\tau_{3}, v^{\prime}}
\end{aligned}
$$


由此我们可以求解哈密顿量的矩阵元. 通过求 解哈密顿量矩阵, 可以得到 PRM 的波函数

$$
|I M\rangle=\sum_{K \varphi} C_{K \varphi}|I M K \varphi\rangle .
$$

在求解过程中, 需要对角化一个大规模的矩阵, 在程序中采用 Lanczos 对角化方法, 其标准化程序 LAPACK 见文献 [76], 其基本原理如下.

(1) 选择一个初始的 Lanczos 矢量 $\left|l a n c_{0}\right\rangle$ 作为最 低本征矢的零阶近似.

(2) 通过

$$
\left|n e w_{p+1}\right\rangle=\hat{H}\left|\operatorname{lanc}_{p}\right\rangle
$$

产生一个新的矢量.

(3) 新的矢量 $\left|n e w_{p+1}\right\rangle$ 与前面计算的 Lanczos 矢 量正交化,

$$
\left|n e w_{p+1}^{\prime}\right\rangle=\left|n e w_{p+1}\right\rangle-\sum_{q=0}^{p}\left|\operatorname{lanc}_{q}\right\rangle\left\langle\operatorname{lanc}_{q} \mid n e w_{p+1}\right\rangle,
$$

然后归一化产生一个新的 Lanczos 矢量

$$
\left.\left.\mid \text { lanc }_{p+1}\right\rangle=\alpha_{p+1} \mid \text { new }^{\prime} p+1\right\rangle .
$$

（4）在这个 Lanczos 基矢上构造哈密顿量矩阵元

$$
\begin{aligned}
& \left.H_{p, p}=\left\langle\operatorname{lanc}_{p}|H| \text { lanc }_{p}\right\rangle=\left\langle\text { lanc }_{p}\right| \text { new }_{p+1}\right\rangle \\
& H_{p+1, p}=\left\langle\text { lanc }_{p+1}|H| \text { lanc }_{p}\right\rangle
\end{aligned}
$$

所有其他矩阵元则为 0 .

(5) 在 $p$ 步迭代之后, 有一个能量矩阵, 形式为

$$
\left(\begin{array}{ccccc}
H_{0,0} & H_{0,1} & 0 & \ldots & 0 \\
H_{1,0} & H_{1,1} & H_{1,2} & \ldots & 0 \\
0 & H_{2,1} & H_{2,2} & \ldots & 0 \\
\vdots & \vdots & \vdots & \vdots & H_{p-1, p} \\
0 & 0 & 0 & H_{p, p-1} & H_{p, p}
\end{array}\right),
$$

$p$ 是相对较小的数, 可以通过标准的方法对角化矩阵, 得到 $p$ 步时的能量本征值.

(6) 重复步骤 2-5直到第 $p$ 步和 $p+1$ 步的能量 本征值的差值小于一个设定的精度, 则认为解收玫.

通过对角化哈密顿量矩阵, 我们得到能量本征 值和本征波函数. 利用波函数, 可以计算电磁跃迁几 率和角动量期待值等, 具体公式见文献 $[66,70]$.

\section{3 多准粒子组态的倾斜轴推转模型}

推转壳模型最早由 Inglis于 1954 年提出 ${ }^{[77]}$, 后 来非常成功地描述了快速转动的原子核. 最初的推 转壳模型主要是沿着惯量主轴转动, 后来 Frauendorf 等人在此基础上推广到倾斜轴推转 $[1,55,78-80]$, 即转 动轴偏离惯量主轴. 三维倾斜轴推转模型广泛用来 描述手征转动. 目前发展了两种版本的倾斜轴推转

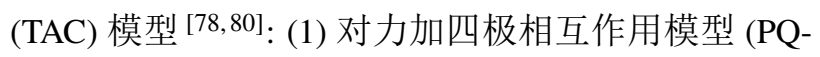
TAC), (2) 壳修正方法 (SCTAC).

一般来讲, PQTAC 更适合描述小形变的原子核, 而 SCTAC 更适合大形变的原子核 ${ }^{[80]}$.

\section{1 对力加四极相互作用模型}

假定 $z$ 轴为转动轴, 倾斜轴推转模型由两体 Routhin 量出发

$$
\hat{H}^{\prime}=\hat{H}-\omega \hat{J}_{z}
$$

它包含转动不变的两体哈密顿量 $H$ 和推转项 $\omega \hat{J}_{z}$. 两 体哈密顿量可采用对力加四极相互作用:

$$
\hat{H}=\hat{H}_{\mathrm{sph}}-\frac{\chi}{2} \sum_{\mu=-2}^{2} \hat{Q}_{\mu}^{+} \hat{Q}_{\mu}-G \hat{P}^{+} \hat{P}-\lambda N
$$

其解释见文献 [75]. 在此模型中我们调节 $\chi$ 的值, 使得从中导出的平均场哈密顿量和 Nilsson 哈密顿

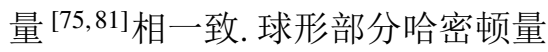

$$
\hat{H}_{\mathrm{sph}}=\sum_{k} \varepsilon_{k} c_{k}^{+} c_{k}
$$

与 Nilsson 哈密顿量具有同样的参数.

对力相互作用由单极对力场定义为

$$
\hat{P}^{+}=\sum_{k>0} c_{k}^{+} c_{\bar{k}}^{+}
$$

其中 $\bar{k}$ 是 $k$ 的时间反演态. 四极相互作用由算符定义 为

$$
\hat{Q}_{\mu}=\sum_{k k^{\prime}} \sqrt{\frac{4 \pi}{5}}\left\langle k\left|r^{2} Y_{2 \mu}\right| k^{\prime}\right\rangle c_{k}^{+} c_{k^{\prime}}
$$

表达式中的粒子包括质子和中子. 
波函数由 Hartree-Fock-Bogoliubov(HFB) 平均场 表达式 |\rangle 近似表示. 忽略交换项, HFB-Routhian 变成

$$
\begin{array}{r}
\hat{h}^{\prime}=\hat{h}_{\mathrm{sph}}-\sum_{\mu=-2}^{2}\left(q_{\mu} \hat{Q}_{\mu}^{+}+q_{\mu}^{*} \hat{Q}_{\mu}\right) \\
-\Delta\left(\hat{P}^{+}+\hat{P}\right)-\lambda \hat{N}-\omega \hat{J}_{z} .
\end{array}
$$

这个自洽方程确定形变势 $q_{\mu}=\chi\left\langle\hat{Q}_{\mu}\right\rangle$ 和对力势 $\Delta=$ $G\langle\hat{P}\rangle$. 化学势 $\lambda$ 由标准条件 $N=\langle\hat{N}\rangle$ 决定. 准粒子算 符为

$$
\alpha_{i}^{+}=\sum_{k}\left(U_{k i} c_{k}^{+}+V_{k i} c_{k}\right)
$$

遵守运动方程

$$
\left[h^{\prime}, \alpha_{i}^{+}\right]=e_{i}^{\prime} \alpha_{i}^{+},
$$

这就是决定准粒子振幅 $U_{k i}$ 和 $V_{k i}$ 的 HFB 本征方

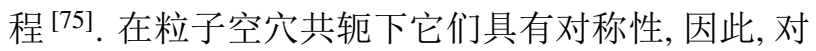
于每一个准粒子 $i$ 就会有相应的共轭态 $i^{+}$

$$
e_{i^{+}}^{\prime}=-e_{i}^{\prime}, \quad U_{k i^{+}}=V_{k i}, \quad V_{k i^{+}}=U_{k i} .
$$

准粒子的宇称是好量子数, 但是一般情况下旋称不 是好量子数.

激发的准粒子组态定义为

$$
\left|i_{1}, i_{2}, \ldots\right\rangle=\alpha_{i_{1}}^{+} \alpha_{i_{2}}^{+} \cdots|0\rangle,
$$

其中 $|0\rangle$ 表示准粒子真空态.

对于任意组态 $\left|i_{1}, i_{2}, \ldots\right\rangle$, HFB 的一系列方程可以 被求解. 对于自洽解, 总 Routhian

$$
E^{\prime}=\left\langle\hat{H}^{\prime}\right\rangle
$$

存在极值

$$
\left.\frac{\partial E^{\prime}}{\partial q_{\mu}}\right|_{\omega}=0,\left.\quad \frac{\partial E^{\prime}}{\partial \Delta}\right|_{\omega}=0 .
$$

总能量作为角动量 $J$ 的函数, 如下

$$
E(J)=E^{\prime}(\omega)+\omega J(\omega),
$$

其中 $J(\omega)=\left\langle\hat{J}_{z}\right\rangle$. 对于自洽解, 总能量也有极值

$$
\left.\frac{\partial E}{\partial q_{\mu}}\right|_{J}=0,\left.\frac{\partial E}{\partial \Delta}\right|_{J}=0 .
$$

这个求解必须在 $J$ 固定时进行. 对于一系列的自洽 解 $|\omega\rangle$, 都遵守正则关系

$$
\frac{\mathrm{d} E^{\prime}}{\mathrm{d} \omega}=-J, \quad \frac{\mathrm{d} E}{\mathrm{~d} J}=\omega .
$$

结果表明对于一个自洽解, 角速度矢量和角动 量矢量必须平行.

$$
\hat{\omega} \| \boldsymbol{J} .
$$

这个结论在内禀坐标系下也成立.

倾斜轴推转模型 ${ }^{[80]}$ 考虑四极张量 $q_{\mu}$ 的主轴和 转动轴 ( $z$ 轴) 不重合的情况, 也就是说, $z$ 轴倾斜于主 轴. 那么,

$$
\mathrm{e}^{-\mathrm{i} \pi \hat{J}_{z}}|\pi, \omega\rangle \neq r|\pi, \omega\rangle
$$

旋称不再是好量子数. 准粒子组态 $|\pi, \omega\rangle$ 描述给定宇 称的 $\Delta I=1$ 的转动带,

通常在四极张量的主轴系求解 TAC 模型. 通过 四极张量所满足的条件来定义内禀坐标系

$$
q_{-1}^{\prime}=q_{1}^{\prime}=0, \quad q_{-2}^{\prime}=q_{2}^{\prime}
$$

主轴表示为 $1,2,3$, 在实验室系下的主轴方向由三个 欧拉角 $\psi, \vartheta, \varphi$ 确定. 两个内禀四极矩 $q_{0}^{\prime}$ 和 $q_{2}^{\prime}$ 决定 势场的形变. 实验室系下的四极矩和内禀四极矩的 关系如下:

$$
\begin{aligned}
q_{\mu}= & D_{\mu 0}^{2}(\psi, \vartheta, \varphi) q_{0}^{\prime}+\left(D_{\mu 2}^{2}(\psi, \vartheta, \varphi)\right. \\
& \left.+D_{\mu-2}^{2}(\psi, \vartheta, \varphi)\right) q_{2}^{\prime}
\end{aligned}
$$

其中 $D_{\mu \nu}^{2}(\psi, \vartheta, \varphi)$ 是 Wigner D 函数 [74].

不同角度 $\psi$ 对应于同一个内禀态, 它们是简并 的. 我们选择 $\psi=0$ 的态. 用 $(\vartheta, \varphi)$ 表示转动轴 $z$ 轴相 对于主轴的位置. 在内禀坐标系下 HFB Routhian 为

$$
\begin{aligned}
\hat{h}^{\prime}= & \hat{h}_{\mathrm{sph}}-q_{0}^{\prime} \hat{Q}_{0}^{\prime}-q_{2}\left(\hat{Q}_{2}^{\prime}+\hat{Q}_{-2}^{\prime}\right)-\Delta\left(\hat{P}^{+}+\hat{P}\right)-\lambda \hat{N} \\
& -\omega\left(\sin \vartheta \sin \varphi \hat{J}_{1}+\sin \vartheta \cos \varphi \hat{J}_{2}+\cos \vartheta \hat{J}_{3}\right) .
\end{aligned}
$$

\section{形状由两个方程}

$$
q_{0}^{\prime}=\kappa\left\langle\hat{Q}_{0}^{\prime}\right\rangle, \quad q_{2}^{\prime}=\kappa\left\langle\hat{Q}_{2}^{\prime}\right\rangle
$$

和方向角 $(\vartheta, \varphi)$ 确定. 总 Routhian 取极值时, 角动量 的期待值和角速度平行, 即

$$
\begin{aligned}
\vec{J} & =\left(\left\langle\hat{J}_{1}\right\rangle,\left\langle\hat{J}_{2}\right\rangle,\left\langle\hat{J}_{3}\right\rangle\right) \| \omega \\
& =(\omega \sin \vartheta \sin \varphi, \omega \sin \vartheta \cos \varphi, \omega \cos \vartheta) .
\end{aligned}
$$




\section{2 壳修正方法}

另一个 $\mathrm{TAC}$ 版本从含 Nilsson 势的 Routhian 出 发,

$$
\begin{aligned}
h^{\prime}= & \sum_{v=1}^{3} \frac{\left(\hat{p}_{v}^{2}+\omega_{v}^{2} \hat{x}_{v}^{2}\right)}{2 M}+\kappa \hat{l}_{v} \hat{s}_{v}+\mu\left(\hat{l}_{v}^{2}-\left\langle\hat{l}_{v}^{2}\right\rangle\right) \\
& -\Delta\left(\hat{P}^{+}+\hat{P}\right)-\lambda \hat{N} \\
& -\omega\left(\sin \vartheta \sin \varphi J_{1}+\sin \vartheta \cos \varphi J_{2}+\cos \vartheta J_{3}\right) .
\end{aligned}
$$

引进拉伸的坐标系 ${ }^{[81]}$, 使得矩阵元 $\left\langle N\left|J_{\mu}\right| N \pm 2\right\rangle$ 可 被忽略, 这是目前的 Nilsson 模型中通常采用的做法. 振荡频率由 Nilsson 形变参数 $\varepsilon$ 和 $\gamma$ 给出,

$$
\omega_{v}=\omega_{0}\left[1-\frac{2}{3} \varepsilon \cos \left(\gamma-\frac{2 \pi v}{3}\right)\right] .
$$

将 Strutinsky壳修正方法用于非转动系统 $E_{0}$ 得到总 的 Routhian. 最小化的总 Routhian 为

$$
\begin{aligned}
& E^{\prime}\left(\omega, \vartheta, \varphi, \varepsilon, \varepsilon_{4}, \gamma, \Delta, \lambda\right) \\
= & E_{L D}\left(\varepsilon, \varepsilon_{4}, \gamma\right)-\widetilde{E}\left(\varepsilon, \varepsilon_{4}, \gamma\right)+h^{\prime}+(2 \Delta-G\langle P\rangle)\langle P\rangle,
\end{aligned}
$$

其中

$$
|\rangle=\left|\omega, \vartheta, \varphi, \varepsilon, \varepsilon_{4}, \gamma, \Delta, \lambda\right\rangle
$$

是上面定义的平均场 Routhian $h^{\prime}\left(\omega, \vartheta, \varphi, \varepsilon, \varepsilon_{4}, \gamma, \Delta, \lambda\right)$ 的一个准粒子态. 能量 $\widetilde{E}$ 是由 Strutinsky 方法 ${ }^{[82]}$ 对单 粒子的能量计算得到, 单粒子能量取为 $h^{\prime}(\omega=0, \vartheta=$ $\left.0, \varphi=0, \varepsilon, \varepsilon_{4}, \gamma, \Delta=0\right)$ 的本征值. $E_{L D}\left(\varepsilon, \varepsilon_{4}, \gamma\right)$ 为液滴 能量的表达式 ${ }^{[81]}$.

对于给定的 $\varepsilon, \varepsilon_{4}, \gamma$, 由公式 (37) 来确定倾斜角. 于是可以寻找 $E^{\prime}\left(\omega, \varepsilon, \varepsilon_{4}, \gamma\right)$ 关于形变参数的最小值. 另一方面, 也可以固定形变参数, 寻找 $E^{\prime}$ 关于倾斜角 $(\vartheta, \varphi)$ 的最小值.

\section{4 结论}

粒子转子模型和倾斜轴推转模型是目前研究原 子核手征转动现象时应用最广泛的两种模型. 本文 详细介绍了两种模型的理论框架及模型的求解. 通 过发展和完善两种理论模型, 将为澄清原子核手征 转动研究中的种种争论奠定基础.

\section{参考文献}

1 Frauendorf S, Meng J. Tilted rotation of triaxial nuclei. Nucl Phys A, 1997, 617: 131-147

2 Starosta K, Koike T, Chiara C J, et al. Chiral doublet structures in Odd-Odd N=75 isotones: Chiral vibrations. Phys Rev Lett, 2001, 86: 971-974

3 Yong-Nam U, Zhu S J, Sakhaee M, et al. Search for the chiral doublet bands in ${ }^{122}$ Cs. J Phys G, 2005, 31: B1-B6

4 Zhu S, Garg U, Nayak B K, et al. A composite chiral pair of rotational bands in the Odd-A nucleus ${ }^{135}$ Nd. Phys Rev Lett, 2003 , 91 : 132501

5 Wang S Y, Liu Y Z, Komatsubara T, et al. Candidate chiral doublet bands in the odd-odd nucleus ${ }^{126}$ Cs. Phys Rev C, $2006,74: 017302$

6 Starosta K, Chiara C J, Fossan D B, et al. Role of chirality in angular momentum coupling forA $\sim 130$ odd-odd triaxial nuclei: ${ }^{132}$ La. Phys Rev C, 2002, 65: 044328

7 Simons A J, Joshi P, Jenkins D G, et al. Evidence for chiral structures in ${ }^{130}$ Cs. J Phys G, 2005, 31: $541-552$

8 Rainovski G, Paul E S, Chantler H J, et al. Planar and aplanar tilted bands in the odd-odd nucleus 132 Cs. J Phys G, 2003, 29: 2763

9 Rainovski G, Paul E S, Chantler H J, et al. Candidate chiral twin bands in the odd-odd nucleus ${ }^{132}$ Cs: Exploring the limits of chirality in the mass $A \sim 130$ region. Phys Rev C, 2003, 68: 024318

10 Mergel E, Petrache C M, Bianco G L, et al. Candidates for chiral doublet bands in ${ }^{136}$ Nd. Eur Phys J A, 2002, 15: 417-420

11 Li X F, Ma Y J, Liu Y Z, et al. Search for the chiral band in the $N=71$ Odd-Odd nucleus ${ }^{126}$ Cs. Chin Phys Lett, 2002, 19: 1779-1781

12 Koike T, Starosta K, Chiara C J, et al. Systematic search of $\pi h_{11 / 2} \otimes v h_{11 / 2}$ chiral doublet bands and role of triaxiality in odd-odd $Z=55$ isotopes: 128,130,132,134Cs. Phys Rev C, 2003, 67: 044319

13 Koike T, Starosta K, Chiara C J, et al. Observation of chiral doublet bands in odd-odd N=73 isotones. Phys Rev C, 2001, 63: 061304(R)

14 Starosta K, Koike T, Chiara C J, et al. Chirality in odd-odd triaxial nuclei. Nucl Phys A, 2001, 682: 375c-386c

15 Hecht A A, Beausang C W, Amro H, et al. Evidence for chiral symmetry breaking in ${ }^{140}$ Eu? Phys Rev C, 2003, 68: 054310

16 Hecht A A, Beausang C W, Zyromski K E, et al. Evidence for chiral symmetry breaking in ${ }^{136}$ Pm and ${ }^{138}$ Eu? Phys Rev C, 2001, 63: 051302(R) 
17 Hartley D J, Riedinger L L, Riley M A, et al. Detailed spectroscopy of the chiral-twin candidate bands in ${ }^{136} \mathrm{Pm}$. Phys Rev C, 2001, 64: 031304

18 Bark R A, Baxter A M, Byrne A P, et al. Candidate chiral band in ${ }^{134}$ La. Nucl Phys A, 2001, 691: 577-598

19 Alcántara-Núñez J A, Oliveira J R B, Cybulska E W, et al. Magnetic dipole and electric quadrupole rotational structures and chirality in ${ }^{105}$ Rh. Phys Rev C, 2004, 69: 024317

20 Zhu S J, Hamilton J H, Ramayya A V, et al. Soft chiral vibrations in ${ }^{106}$ Mo. Eur Phys J A, 2005, 25: 459-462

21 Vaman C, Fossan D B, Koike T, et al. Chiral degeneracy in triaxial ${ }^{104}$ Rh. Phys Rev Lett, 2004, 92: 032501

22 Timá J, Koike T, Pietralla N, et al. High-spin structure of ${ }^{105} \mathrm{Ag}$ : Search for chiral doublet bands. Phys Rev C, 2007, 76: 024307

23 Timár J, Vaman C, Starosta K, et al. Role of the core in degeneracy of chiral candidate band doubling: ${ }^{103}$ Rh. Phys Rev C, 2006, 73: 011301(R)

24 Timár J, Joshi P, Starosta K, et al. Experimental evidence for chirality in the odd-A ${ }^{105}$ Rh. Phys Lett B, 2004, 598: 178-187

25 Joshi P, Carpenter M P, Fossan D B, et al. Effect of $\gamma$ softness on the stability of chiral geometry: Spectroscopy of ${ }^{106}$ Ag. Phys Rev Lett, 2007, 98: 102501

26 Joshi P, Wilkinson A R, Koike T, et al. First evidence for chirality in Tc isotopes: Spectroscopy of ${ }^{100}$ Tc. Eur Phys J A, 2005, 24: $23-29$

27 Joshi P, Jenkins D G, Raddon P M, et al. Stability of chiral geometry in the odd-odd Rh isotopes: Spectroscopy of ${ }^{106}$ Rh. Phys Lett B, 2004, 595: $135-142$

28 He C Y, Zhu L H, Wu X G, et al. Experimental study on chirality in ${ }^{106}$ Ag. High Energy Phys Nucl Phys, 2006, 30: 166-168

29 Lawrie E A, Vymers P A, Lawrie J J, et al. Possible chirality in the doubly-odd ${ }^{198}$ Tl nucleus: Residual interaction at play. Phys Rev C, 2008, 78: 021305(R)

30 Balabanski D L, Danchev M, Hartley D J, et al. Possible assignment of chiral twin bands in ${ }^{188}$ Ir. Phys Rev C, 2004, 70: 044305

31 Wang S Y, Qi B, Liu L, et al. The first candidate for chiral nuclei in the A $\sim 80$ mass region: ${ }^{80}$ Br. Phys Lett B, 2011, 703: 40-45

32 Tonev D, de Angelis G, Petkov P, et al. Transition probabilities in ${ }^{134}$ Pr: A test for chirality in nuclear systems. Phys Rev Lett, 2006, $96: 052501$

33 Tonev D, de Angelis G, Brant S, et al. Question of dynamic chirality in nuclei: The case of ${ }^{134}$ Pr. Phys Rev C, 2007, 76: 044313

34 Petrache C M, Hagemann G B, Hamamoto I, et al. Risk of misinterpretation of nearly degenerate pair bands as chiral partners in nuclei. Phys Rev Lett, 2006, 96: 112502

35 Grodner E, Srebrny J, Pasternak A A, et al. ${ }^{128}$ Cs as the best example revealing chiral symmetry breaking. Phys Rev Lett, 2006, $97:$ 172501

36 Mukhopadhyay S, Almehed D, Garg U, et al. From chiral vibration to static chirality in ${ }^{135}$ Nd. Phys Rev Lett, 2007, $99: 172501$

37 Grodner E, Sankowska I, Morek T, et al. Partner bands of ${ }^{126} \mathrm{Cs} —$ first observation of chiral electromagnetic selection rules. Phys Lett B, 2011, 703: 46-50

38 Tonev D, Yavahchova M S, Goutev N, et al. Candidates for twin chiral bands in ${ }^{102}$ Rh. Phys Rev Lett, 2014, 112: 052501

39 Rather N, Datta P, Chattopadhyay S, et al. Exploring the origin of degenerate doublet bands in ${ }^{106}$ Ag. Phys Rev Lett, 2014, 112: 202503

40 Lieder E O, Lieder R M, Bark R A, et al. Resolution of chiral conundrum in ${ }^{106}$ Ag: Doppler-shift lifetime investigation. Phys Rev Lett, 2014, 112: 202502

41 Meng J, Peng J, Zhang S Q, et al. Possible existence of multiple chiral doublets in ${ }^{106}$ Rh. Phys Rev C, 2006, 73: 037303

42 Peng J, Sagawa H, Zhang S Q, et al. Search for multiple chiral doublets in rhodium isotopes. Phys Rev C, 2008, 77: 024309

43 Yao J M, Qi B, Zhang S Q, et al. Candidate $\mathrm{M} \chi \mathrm{D}$ nucleus ${ }^{106} \mathrm{Rh}$ in triaxial relativistic mean-field approach with time-odd fields. Phys Rev $\mathrm{C}$, 2009, 79: 067302

44 Li J, Zhang S Q, Meng J. Multiple chiral doublet candidate nucleus ${ }^{105} \mathrm{Rh}$ in a relativistic mean-field approach. Phys Rev C, 2011, 83: 037301

45 Ayangeakaa A D, Garg U, Anthony M D, et al. Evidence for multiple chiral doublet bands in ${ }^{133}$ Ce. Phys Rev Lett, 2013, $110: 172504$

46 Kuti I, Chen Q B, Timár J, et al. Multiple chiral doublet bands of identical configuration in ${ }^{103}$ Rh. Phys Rev Lett, 2014, 113: 032501

47 Meng J. Is there chirality in atomic nuclei? Physics, 2009, 38: 108-112 [孟杰. 原子核是否存在手性. 物理, 2009, 38: 108-112]

48 Meng J, Qi B, Zhang S Q, et al. Chiral symmetry in atomic nuclei. Mod Phys Lett A, 2008, 23: 2560-2567

49 Meng J. New aspects of chiral symmetry breaking in atomic nucleus. J Phys-Conf Ser, 2010, 205: 012030

50 Meng J, Zhang S Q. Open problems in understanding the nuclear chirality. J Phys G-Nucl Part Phys, 2010, 37: 064025

51 Meng J, Chen Q B, Zhang S Q. Chirality in atomic nuclei. Int J Modern Phys E-Nucl Phys, 2014, 23: 11: 1430016

52 Dar W A, Sheikh J A, Bhat G H, et al. Microscopic study of doublet bands in odd-odd A 100 nuclei. Nucl Phys A, 2015, 933: 123-134

53 Chen Q B, Zhang S Q, Zhao P W, et al. Collective Hamiltonian for chiral modes. Phys Rev C, 2013, 87: 024314

54 Dimitrov V I , Frauendorf S, Dönau F. Chirality of nuclear rotation. Phys Rev Lett, 2000, 84: 5732 
55 Frauendorf S. Spontaneous symmetry breaking in rotating nuclei. Rev Mod Phys, 2001, 73: 463

56 Olbratowski P, Dobazewski J, Dudek J, et al. Critical frequency in nuclear chiral rotation. Phys Rev Lett, 2004, 93: 052501

57 Olbratowski P, Dobazewski J, Dudek J. Search for the Skyrme-Hartree-Fock solutions for chiral rotation in $N=75$ isotones. Phys Rev C, 2006 , 73: 054308

58 Zhao P W, Zhang S Q, Peng J, et al. Novel structure for magnetic rotation bands in ${ }^{60}$ Ni. Phys Lett B, 2011, 699: 181-186

59 Zhao P W, Peng J, Liang H Z, et al. Antimagnetic rotation band in nuclei: A microscopic description. Phys Rev Lett, 2011, 107: 122501

60 Zhao P W, Peng J, Liang H Z, et al. Covariant density functional theory for antimagnetic rotation. Phys Rev C, 2012, 85: 054310

61 Zhang P, Qi B, Wang S Y. Competition between antimagnetic and core rotation in ${ }^{109} \mathrm{Cd}$ within covariant density functional theory. Phys Rev C, 2014, 89: 047302

62 Peng J, Zhao P W. Magnetic and antimagnetic rotation in ${ }^{110} \mathrm{Cd}$ within tilted axis cranking relativistic mean-field theory. Phys Rev C, 2015, 91 : 044329

63 Peng J, Meng J, Zhang S Q. Description of chiral doublets in $A \sim 130$ nuclei and the possible chiral doublets in $A \sim 100$ nuclei. Phys Rev C, 2003, 68: 044324

64 Koike T, Starosta K, Hamamoto I. Chiral bands, dynamical spontaneous symmetry breaking, and the selection rule for electromagnetic transitions in the chiral geometry. Phys Rev Lett, 2004, 93: 172502

65 Qi B, Zhang S Q, Wang S Y, et al. Examining B(M1) staggering as a fingerprint for chiral doublet bands. Phys Rev C, 2009, 79: 041302(R)

66 Zhang S Q, Qi B, Wang S Y, et al. Chiral bands for a quasi-proton and quasi-neutron coupled with a triaxial rotor. Phys Rev C, 2007, 75: 044307

67 Wang S Y, Qi B, Sun D P. Theoretical study of positive-parity doublet bands in ${ }^{124}$ Cs. Phys Rev C, 2010, 82: 027303

68 Wang S Y, Zhang S Q, Qi B, et al. Description of $\pi g_{9 / 2} \otimes v h_{11 / 2}$ doublet bands in ${ }^{106} \mathrm{Rh}$. Phys Rev C, 2008, 77: 034314

69 Wang S Y, Zhang S Q, Qi B, et al. Doublet bands in ${ }^{126}$ Cs in the triaxial rotor model coupled with two quasiparticles. Phys Rev C, 2007, 75 : 024309

70 Qi B, Zhang S Q, Meng J, et al. Chirality in odd-A nucleus ${ }^{135} \mathrm{Nd}$ in particle rotor model. Phys Lett B, 2009, 675: 175

71 Qi B, Zhang S Q, Wang S Y, et al. Chirality in odd-A Rh isotopes within the triaxial particle rotor model. Phys Rev C, 2011, 83: 034303

72 Qi B, Jia H, Zhang N B, et al. Possible multiple chiral doublet bands in ${ }^{107}$ Ag. Phys Rev C, 2013, 88: 027302

73 Ayangeakaa A D, Garg U, Anthony M D, et al. Evidence for multiple chiral doublet bands in ${ }^{133}$ Ce. Phys Rev Lett, 2013, $110: 172504$

74 Bohr A, Mottelson B. Nuclear Structure. New York: Benjamin, 1975

75 Ring P, Shuck P. The Nuclear Many-body Problem. New York: Springer-Verlag, 1980

76 Anderson E, Bai Z, Bischof C, et al. LAPACK Users' Guide. Philadelphia: SIAM, 1995

77 Inglis D R. Particle derivation of nuclear rotation properties associated with a surface wave. Phys Rev, 1954, 98: 1059

78 Frauendorf S. Tilted cranking. Nucl Phys A, 1993, 557: 259c-276c

79 Dönau F, Frauendorf S, Vogel O, et al. Tilted four-quasiparticle bands in even-even Xe and Ba nuclei. Nucl Phys A, 1995, 584: 241-255

80 Frauendorf S. Description of multi-quasiparticle bands by the tilted axis cranking model. Nucl Phys A, 2000, 677: 115-170

81 Nilsson S G, Ragnarsson I. Shapes and Shells in Nuclear Structure. Cambridge: Cambridge University Press, 1995

82 Strutinsky V M. Shell effects in nuclear masses and deformation energies. Nucl Phys A, 1967, 95: 420-422 


\title{
Chiral rotational models with multi-quasiparticle configuration
}

\author{
QI Bin* \& JIA Hui \\ Shandong Provincial Key Laboratory of Optical Astronomy and Solar-Terrestrial Environment, School of Space Science and Physics, and \\ Institute of Space Sciences, Shandong University, Weihai 264209, China
}

The chiral rotation in atomic nuclei has attracted significant attention in recent years. This paper introduces the particle rotor model and the titled axis cranking model, which are used to analyze the chiral rotation with multi-quasiparticle configuration. For the particle rotor model, the basis space and the method to solve the model are discussed in detail: by considering the single-particle states of valence nucleons and the selection rule of angular momentum, one can construct the strong coupling basis which is symmetrized under the point group $D_{2}$; the dimension of the basis space will be very large when several valence nucleons are included, thus the Lancozos method is adopted to diagonalize the large scale hamiltonian matrix. For the titled axis cranking model, two versions based on the pairing plus quadrupole model and the shell correction method are introduced respectively: the cranking Hartree-Fock-Bogoliubov equations are given starting with the two-body Routhian; the 3-dimensional tilted axis cranking solutions are discussed in the intrinsic principal axes frame, and the vector of the angular velocity is parallel with the vector of the expectation values of the angular momentum components when the self-consistent solution is obtained. By summarizing and developing further the two models, it will be helpful to clarify the debates in the understanding of the nuclear chiral rotation.

chiral rotation, particle rotor model, titled axis cranking model, multi-quasiparticle configuration

PACS: 21.60.Ev, 21.10.Re, 23.20.Lv

doi: 10.1360/SSPMA2015-00362 